\title{
Asymmetric Metasurfaces with High- $Q$ Resonances Governed by Bound States in the Continuum
}

\author{
Kirill Koshelev, ${ }^{1,2}$ Sergey Lepeshov, ${ }^{2}$ Mingkai Liu, ${ }^{1}$ Andrey Bogdanov, ${ }^{2}$ and Yuri Kivshar ${ }^{1,2}$ \\ ${ }^{1}$ Nonlinear Physics Centre, Australian National University, Canberra ACT 2601, Australia \\ ${ }^{2}$ ITMO University, St. Petersburg 197101, Russia
}

(Received 28 June 2018; published 9 November 2018)

\begin{abstract}
We reveal that metasurfaces created by seemingly different lattices of (dielectric or metallic) meta-atoms with broken in-plane symmetry can support sharp high- $Q$ resonances arising from a distortion of symmetry-protected bound states in the continuum. We develop a rigorous theory of such asymmetric periodic structures and demonstrate a link between the bound states in the continuum and Fano resonances. Our results suggest the way for smart engineering of resonances in metasurfaces for many applications in nanophotonics and metaoptics.
\end{abstract}

DOI: 10.1103/PhysRevLett.121.193903

Metasurfaces have attracted a lot of attention in recent years due to novel ways they provide for wave front control, advanced light focusing, and ultrathin optical elements [1]. Recently, metasurfaces based on high-index resonant dielectric nanoparticles [2] have emerged as essential building blocks for various functional metaoptics devices [3] due to their low intrinsic losses and unique capabilities for controlling the propagation and localization of light. A key concept underlying the specific functionalities of many metasurfaces is the use of constituent elements with spatially varying optical properties and optical response characterized by high quality factors $(Q$ factors) of the resonances.

Many interesting phenomena have been demonstrated for metasurfaces composed of arrays of meta-atoms with broken in-plane inversion symmetry (see Fig. 1), which all demonstrate the excitation of high- $Q$ resonances for the normal incidence of light. Examples include imaging-based molecular bar coding with pixelated dielectric metasurfaces [4] and polarization-induced chirality in metamaterials [5], which both involve asymmetric pairs of tilted bars [see Fig. 1(a)], trapped modes in arrays of dielectric nanodisks with asymmetric holes [6] [see Fig. 1(b)], sharp trappedmode resonances in plasmonic and dielectric split-ring structures [7,8] [see Fig. 1(c)], broken-symmetry Fano metasurfaces for enhanced nonlinear effects $[9,10]$ [see Fig. 1(d)], tunable high- $Q$ Fano resonances in plasmonic and dielectric metasurfaces [11-13] [see Fig. 1(e)], trapped light and metamaterial-induced transparency in arrays of square split-ring resonators [14,15] shown in Fig. 1(f). Here, we reveal that all such seemingly different structures can be unified by a general concept of bound states in the continuum, and we prove rigorously their link to the Fano resonances.

Bound states in the continuum (BIC) represent a general wave phenomenon observed in acoustics, hydrodynamics, and optics [16-19]. Originally, this concept appeared in quantum mechanics [20], but later it was explained in terms of destructive interference when the coupling constants with all radiating waves vanish accidentally via continuous

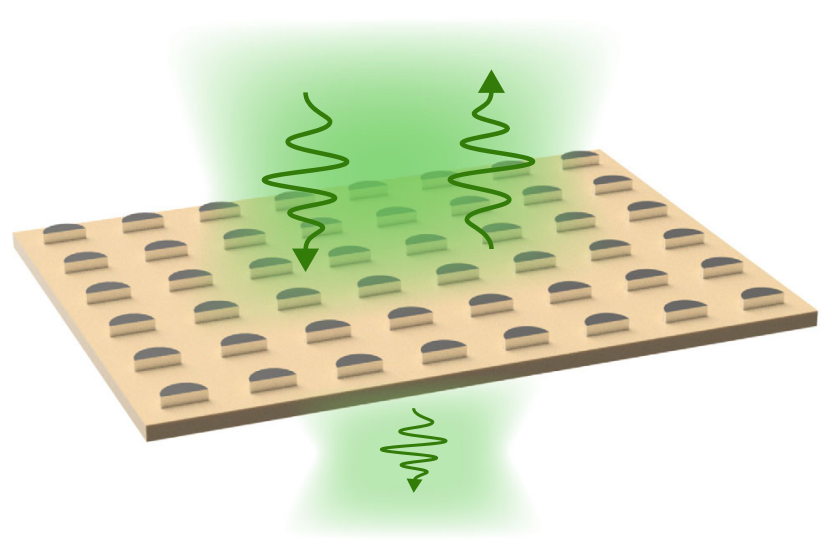

(a)

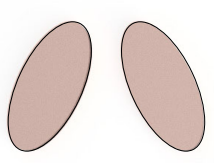

(d)

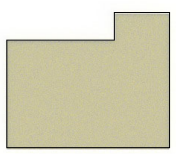

(b)

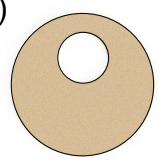

(e)

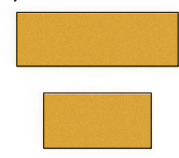

(c)

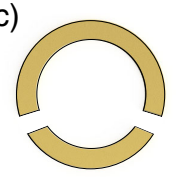

(f)

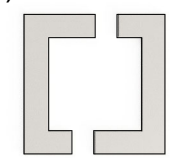

FIG. 1. Top: Schematic for the scattering of light by a metasurface. Bottom: Designs of the unit cells of asymmetric metasurfaces with a broken in-plane inversion symmetry of constituting meta-atoms supporting sharp resonances, as considered in Refs. [4-15]. 

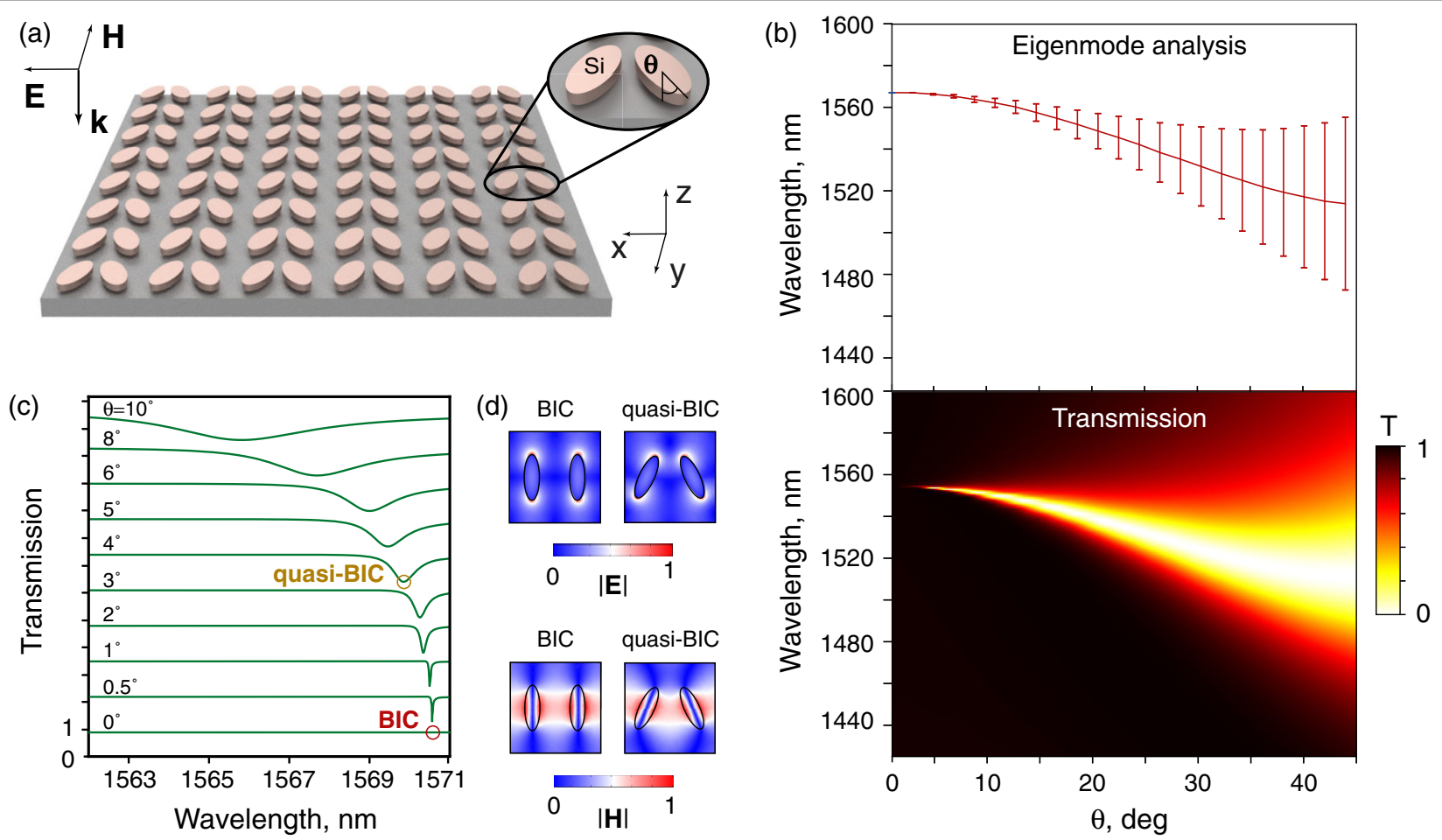

FIG. 2. (a) A square lattice of tilted silicon-bar pairs with a design of the unit cell. Parameters: the period is $1320 \mathrm{~nm}$, bar semiaxes are 330 and $110 \mathrm{~nm}$, height is $200 \mathrm{~nm}$, distance between bars is $660 \mathrm{~nm}$. (b) Eigenmode spectra and transmission spectra with respect to pump wavelength and angle $\theta$. Error bars show the magnitude of the mode inverse radiation lifetime. (c) Evolution of the transmission spectra vs angle $\theta$. Spectra are relatively shifted by 1.5 units. (d) Distribution of the electric and magnetic fields for both BIC and quasi-BIC.

tuning of parameters [21]. Such a mechanism is known as the Fridrich-Wintgen scenario. If the coupling constants vanish due to symmetry, such BIC are called symmetry protected.

A true BIC is a mathematical object with an infinite value of the $Q$ factor and vanishing resonance width, and it can exist only in ideal lossless infinite structures or for extreme values of parameters [22-24]. In practice, BIC can be realized as a quasi-BIC, also known as a supercavity mode [25], when both the $Q$ factor and resonance width become finite [26,27]. Nevertheless, the BIC-inspired mechanism of light localization makes possible to realize high- $Q$ quasiBICs in optical cavities and photonic crystal slabs [19,23,25,28], coupled optical waveguides [29-32], and even isolated subwavelength dielectric particles [33].

Here, we reveal that sharp spectral resonances recently reported and even observed for various types of seemingly different (plasmonic and dielectric) metasurfaces originate from the powerful physics of BIC as a result of distortion of the symmetry-protected bound state in the continuum. We demonstrate that true BICs transform into quasi-BICs when the in-plane inversion symmetry of a unit cell becomes broken, and we derive the universal formula for the $Q$ factor as a function of the asymmetry parameter. We develop an analytical approach to describe light scattering by arrays of meta-atoms based on the explicit expansion of the Green's function of open systems into eigenmode contributions, and demonstrate rigorously that reflection and transmission coefficients are linked to the conventional Fano formula. We prove that the Fano parameter becomes ill-defined at the BIC condition which corresponds to a collapse of the Fano resonance $[34,35]$.

To gain a deeper insight into the physics of BICs in metasurfaces with in-plane asymmetry, we focus on one of the examples recently suggested for biosensing [4], a square array of tilted silicon-bar pairs shown in Fig. 2(a). For our analysis, we consider a homogenous background with permittivity 1 , and calculate both the eigenmodes and transmission spectra, as shown in Fig. 2(b). An asymmetry parameter here is the angle $\theta$ between the $y$ axis and the long axis of the bar. The ideal (lossless and infinite) structure supports a symmetry-protected BIC [36] at $\theta=0^{\circ}$. Such an ideal BIC is unstable against perturbations that break the inplane inversion symmetry $(x, y) \rightarrow(-x,-y)$, and it transforms into a quasi-BIC with a finite $Q$ factor.

The eigenmode and transmission spectra are shown in Fig. 2(b) as functions of $\theta$, where $t$ is the amplitude of the transmitted wave, and $T=|t|^{2}$ is the transmittance. We observe that BIC with infinite $Q$ factor at $\theta=0^{\circ}$ transforms into high- $Q$ quasi-BIC whose radiation loss grows with $\theta$. The detailed transmission spectra shown in Fig. 2(c) exhibit a narrow dip that vanishes when the pair becomes symmetric, 
which confirms the results of the eigenmode analysis. Figure 2(d) demonstrates the similarity of the electric and magnetic fields in BIC and quasi-BIC within a unit cell. Our analysis shows that BIC is characterized by a polarization vortex in the reciprocal space with a unity topological charge (see details in the Supplemental Material [37]).

We analyze the transmission spectra of the metasurfaces and prove rigorously that they can be described by the classical Fano formula, and the observed peak positions and linewidths correspond exactly to the real and imaginary parts of the eigenmode frequencies. While the analytical solution of Maxwell's equations does not exist, the description of the transmission $T$ with the Fano formula is still widely used by introducing the Fano parameter phenomenologically [42]. Here, we demonstrate the explicit correspondence between the Fano line shape of the transmission spectra and properties of the eigenmode spectra, discussed previously only for special cases $[18,23,43]$.

To derive an analytical expression for light transmission, we expand the transmitted field amplitude into a sum of independent terms where each term corresponds to an eigenmode of the photonic structure. This becomes possible by applying the recently developed procedure allowing for rigorous characterization of the Green's function and, therefore, the eigenmode spectrum of open optical resonators [44]. The eigenmodes of a metasurface are treated as self-standing electromagnetic excitations with a complex spectrum describing both the resonant frequencies $\omega_{0}$ and inverse lifetimes $\gamma$. Straightforward but rather cumbersome calculations (see Supplemental Material [37]) reveal that the frequency dependence of the transmission $T$ is described rigorously by the Fano formula, and the Fano parameters are expressed explicitly through the material and geometrical parameters of the metasurface and dimensionless frequency $\Omega=2\left(\omega-\omega_{0}\right) / \gamma$,

$$
T(\omega)=\frac{T_{0}}{1+q^{2}} \frac{(q+\Omega)^{2}}{1+\Omega^{2}}+T_{\mathrm{bg}}(\omega) .
$$

Here $q$ is the Fano asymmetry parameter, $T_{\mathrm{bg}}$ and $T_{0}$ describe the background contribution of nonresonant modes to the resonant peak amplitude and the offset, respectively (see the explicit expressions in the Supplemental Material [37]). Importantly, the frequency dependence of $q, T_{0}$, and $T_{\mathrm{bg}}$ is smooth, and it can be neglected, that is valid when the neighboring resonances do not overlap spectrally with the resonance under study. Remarkably, the exact formula shows that the parameter $q$ in Eq. (1) becomes ill-defined for a true BIC thus corresponding to a collapse of the Fano resonance when any features in the transmission spectra disappear, and the resonant mode is transformed into a "dark mode," which does not manifest itself in scattering spectra.

Next, we describe analytically the behavior of the radiative $Q$ factor of the quasi-BIC as a function of $\theta$ shown in Figs. 2(b) and 2(c). We consider the radiation losses as a perturbation which is a natural approximation valid when $\theta$ remains relatively small. Then, the inverse radiation lifetime $\gamma_{\mathrm{rad}}$ can be calculated as a sum of radiation losses into all open radiation channels. We focus on quasi-BICs with the frequencies below the diffraction limit for which only open radiation channels represent the zeroth-order diffraction. Then $\gamma_{\text {rad }}$ of quasi-BIC takes the form (see Supplemental Material [37]),

$$
\begin{aligned}
\frac{\gamma_{\mathrm{rad}}}{c} & =\left|D_{x}\right|^{2}+\left|D_{y}\right|^{2}, \\
D_{x, y} & =-\frac{k_{0}}{\sqrt{2 S_{0}}}\left(p_{x, y} \mp \frac{1}{c} m_{y, x}+\frac{i k_{0}}{6} Q_{x z, y z}\right) .
\end{aligned}
$$

Here, $k_{0}=\omega_{0} / c, S_{0}$ is the unit cell area, $D_{x, y}$ are the coupling amplitudes between quasi-BIC and zero-order diffraction channels of two orthogonal polarizations, and $\mathbf{p}$, $\mathbf{m}$, and $\hat{\mathbf{Q}}$ are the normalized electric dipole, magnetic dipole, and electric quadrupole moments per unit cell.

Equations 2(a) and 2(b) show that a symmetry mismatch for a true BIC leads to zero $D_{x, y}$ and vanishing radiation losses [45]. In other words, the electric field components $E_{x}$ and $E_{y}$ of BIC are odd with respect to the inversion of coordinates $(x, y) \rightarrow(-x,-y)$, so that $\gamma_{\mathrm{rad}}=0$. For quasiBICs, we perform straightforward transformations of Eqs. 2(a) and 2(b) (see Supplemental Material [37]) to show that the radiative quality factor $Q_{\mathrm{rad}}=\omega_{0} / \gamma_{\mathrm{rad}}$ depends on $\theta$ as

$$
Q_{\mathrm{rad}}(\theta)=Q_{0}[\alpha(\theta)]^{-2},
$$

where $\alpha=\sin \theta$ and $Q_{0}$ is a constant determined by the metasurface design, being independent on $\theta$. In general, Eq. (3) remains valid for metasurfaces placed on a substrate as long as the quasi-BIC frequency is below the diffraction limit of the substrate [27].

Next, we demonstrate that the quadratic dependence defined by Eq. (3) represents a universal behavior for the $Q$ factor of a quasi-BIC as a function of the asymmetry parameter for all studied metasurfaces with brokensymmetry meta-atoms. We introduce the generalized asymmetry parameter $\alpha$, which has distinct definitions for different structures but takes values between 0 and 1 . We derive Eq. (3) using the second-order perturbation theory for open electromagnetic systems and confirm the result by independent calculations of the eigenmode and reflectance spectra for all designs presented in Figs. 1(b)-1(f) (see Supplemental Material [37]). Since plasmonic metasurfaces possess significant absorption, we extract the bare radiative $Q$ factor by evaluating the inverse radiative lifetime $\gamma_{\text {rad }}$ being a difference between the total inverse lifetime $\gamma$ and nonradiative damping rate evaluated at $\alpha=0$.

Figure 3(a) shows a direct comparison of the values of the radiative $Q$ factor of quasi-BICs as functions of the asymmetry parameter $\alpha$ for dielectric and plasmonic 


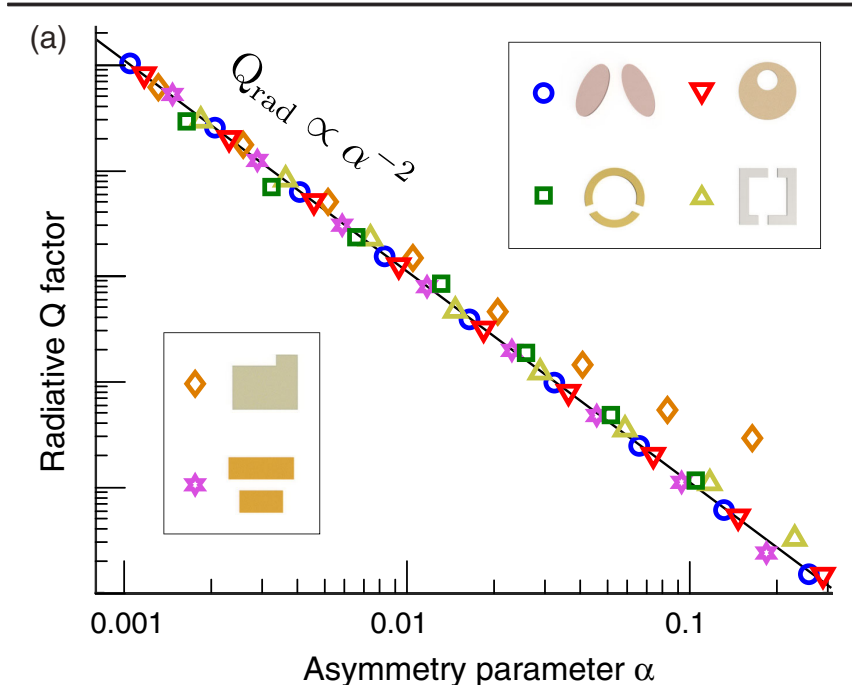

(b)

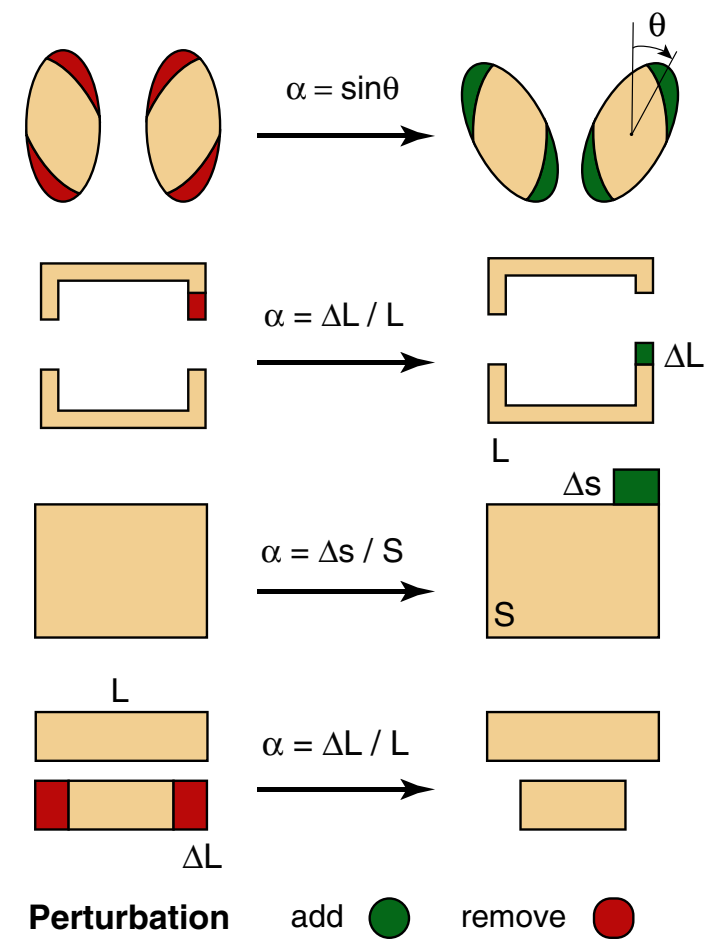

FIG. 3. Effect of the in-plane asymmetry on the radiative $Q$ factor of quasi-BICs. (a) Dependence of the $Q$ factor on the asymmetry parameter $\alpha$ for all designs of symmetry-broken metaatoms shown in Figs. 1(a)-1(f) (log-log scale). (b) Definitions of the asymmetry parameter $\alpha$ for different metasurfaces.

metasurfaces with various broken-symmetry meta-atoms in the unit cell. All curves represent the results of our direct numerical simulations being shifted relatively in the vertical direction to originate from the same point. As can be noticed from Fig. 3(a), for small values of $\alpha$ the behavior of $Q_{\text {rad }}$ for all metasurfaces is clearly inverse quadratic. Importantly, for most of the structures the law $\alpha^{-2}$ is valid beyond the applicability limits of the perturbation theory. Figure 3(b) defines the asymmetry parameter $\alpha$ for different metasurface designs.

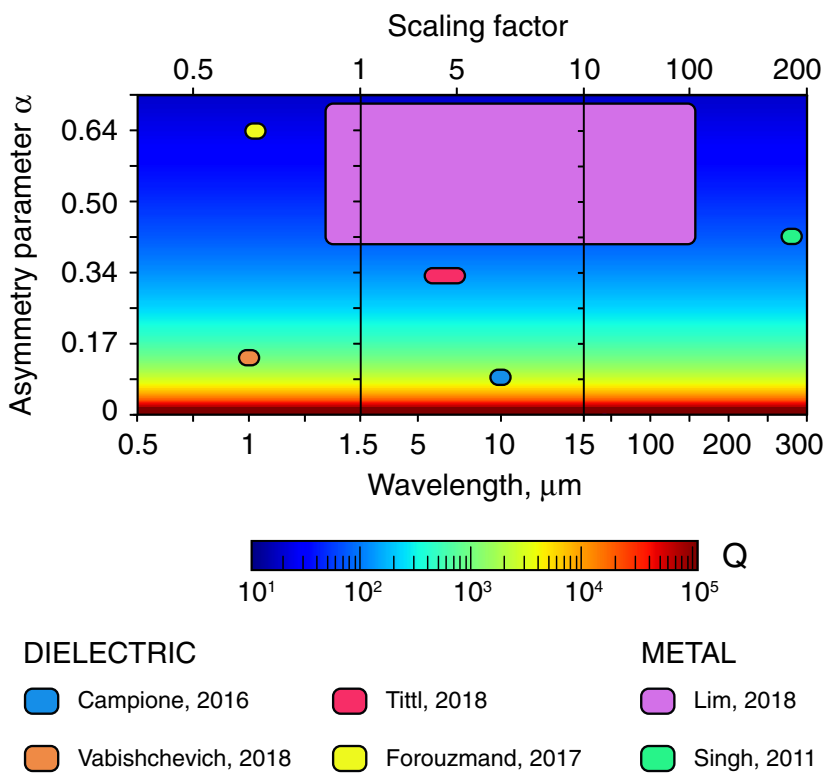

FIG. 4. Map of operating wavelengths and quality factors for silicon metasurfaces with tilted-bar pairs for varying orientation of the bars $(\alpha=\sin \theta)$ and scaling factor. The radiative part of the total $Q$ factor is evaluated using Eq. (3), the nonradiative part is taken equal $10^{5}$. All calculations are confirmed by direct numerical simulations with realistic dispersion of silicon (see Supplemental Material [37]). The geometric scaling factor is shown in the upper horizontal axis. Colored rectangles correspond to the structures considered in the previous studies, see Fig. 1.

The quadratic scalability of the $Q$ factor of quasi-BICs, combined with linear scalability of Maxwell's equations, suggests a straightforward way of smart engineering of photonic structures with the properties on demand. As an example, we focus on a design of tilted silicon-bar pairs and suggest a very simple algorithm for a design of metasurfaces with a wide range of $Q$ factors and operating wavelengths ranging from visible to THz. First, since the refractive index dispersion for silicon is relatively weak, we can tune the operating wavelength from 0.5 to $300 \mu \mathrm{m}$ by a linear geometric scaling of the structure in all dimensions. Second, we can control the mode radiative $Q$ factor in a wide range of parameters by changing the asymmetry parameter $\alpha=\sin \theta$ according to Eq. (3). The total $Q$ factor of quasi-BIC is limited by absorption, which can be estimated as $\operatorname{Re}(\varepsilon) / \operatorname{Im}(\varepsilon)$. For silicon in the frequency range from near-IR to $\mathrm{THz}$, the $Q$ factor can exceed $10^{5}$.

Using this approach, we calculate the dependence of the total $Q$ factor of quasi-BIC vs the operating wavelength and asymmetry parameter for a square lattice of tilted siliconbar pairs, and summarize our results in Fig. 4. For each data point of this map, we perform only one numerical simulation with fixed material parameters to obtain the value of $Q_{0}$ required for Eq. (3), and then employ the advantages of the geometrical scaling combined with rotation of bars. 
We observe that a variety of the required $Q$ factors can be achieved in a broad range from 10 up to $10^{5}$ for each wavelength domain, by using the same material and design. The colored rectangles show the range of the $Q$ factors and operating wavelengths related to the previous studies of metasurfaces with symmetry-broken meta-atoms $[4,8-10,13,15]$. We verify the applicability of the proposed analytical approach by three-dimensional electromagnetic simulations taking into account the silicon dispersion and by using the COMSOL finite-element method, the results are summarized in the Supplemental Material [37]. We observe a good agreement, and thus justify the validity of our analytical scaling method.

We believe that our approach based on the BIC concept can describe many other cases of symmetry-broken metasurfaces and photonic-crystal slabs studied earlier by other approaches and different applications in mind [46-50]. Also, our approach can be helpful to get a deeper physical insight into many other problems in optics, including the existence of dark states in dielectric inclusions coupled to the external waves by small nonresonant metallic antennas [51] and electromagnetically induced transparency [52,53]. We argue that almost any problem involving the so-called "dark states" may find its rigorous formulation in the framework of the theory of BIC resonances.

In conclusion, we have revealed that high- $Q$ resonances recently observed in metasurfaces composed of dissimilar meta-atoms with broken in-plane symmetry are closely associated with the physics of bound states in the continuum. We have proven a direct link between peculiarities in the transmission spectra and Fano resonances, and demonstrated analytically a large variation of the $Q$ factors that can be realized with changing the unit-cell asymmetry, paving the way towards smart engineering of resonances in metaoptics for nanolasers, light-emitting metasurfaces, nonlinear nanophotonics, optical sensors, and ultrafast active metadevices.

The authors acknowledge financial support from the Australian Research Council, the Alexander von Humboldt Stiftung, and the Russian Science Foundation (18-72-10140), and also thank H. Altug, H. Atwater, F. Capasso, A. Krasnok, S. Kruk, W. Liu, Th. Pertsch, M. Rybin, R. Singh, I. Staude, V. Tuz, and N. Zheludev for useful discussions and suggestions. K. K. and A. B. acknowledge a support from the Foundation for the Advancement of Theoretical Physics and Mathematics "BASIS" (Russia).

[1] N. Yu and F. Capasso, Flat optics with designer metasurfaces, Nat. Mater. 13, 139 (2014).

[2] A. I. Kuznetsov, A. E. Miroshnichenko, M. L. Brongersma, Y.S. Kivshar, and B. Lukyanchuk, Optically resonant dielectric nanostructures, Science 354, aag2472 (2016).

[3] S. Kruk and Y. Kivshar, Functional meta-optics and nanophotonics governed by Mie resonances, ACS Photonics 4, 2638 (2017).
[4] A. Tittl, A. Leitis, M. Liu, F. Yesilkoy, D. Y. Choi, D. N. Neshev, Y.S. Kivshar, and H. Altug, Imaging-based molecular barcoding with pixelated dielectric metasurfaces, Science 360, 1105 (2018).

[5] M. Liu, D. A. Powell, R. Guo, I. V. Shadrivov, and Y. S. Kivshar, Polarization-induced chirality in metamaterials via optomechanical interaction, Adv. Opt. Mater. 5, 1600760 (2017).

[6] V. R. Tuz, V. V. Khardikov, A. S. Kupriianov, K. L. Domina, S. Xu, H. Wang, and H.-B. Sun, High-quality trapped modes in all-dielectric metamaterials, Opt. Express 26, 2905 (2018).

[7] V. A. Fedotov, M. Rose, S. L. Prosvirnin, N. Papasimakis, and N. I. Zheludev, Sharp Trapped-Mode Resonances in Planar Metamaterials with a Broken Structural Symmetry, Phys. Rev. Lett. 99, 147401 (2007).

[8] A. Forouzmand and H. Mosallaei, All-dielectric $C$-shaped nanoantennas for light manipulation: Tailoring both magnetic and electric resonances to the desire, Adv. Opt. Mater. 5, 1700147 (2017).

[9] S. Campione, S. Liu, L. I. Basilio, L. K. Warne, W. L. Langston, T. S. Luk, J. R. Wendt, J. L. Reno, G. A. Keeler, I. Brener, and M. B. Sinclair, Broken symmetry dielectric resonators for high quality factor Fano metasurfaces, ACS Photonics 3, 2362 (2016).

[10] P. P. Vabishchevich, S. Liu, M. B. Sinclair, G. A. Keeler, G. M. Peake, and I. Brener, Enhanced second-harmonic generation using broken symmetry III-V semiconductor Fano metasurfaces, ACS Photonics 5, 1685 (2018).

[11] A. B. Evlyukhin, S. I. Bozhevolnyi, A. Pors, M. G. Nielsen, I. P. Radko, M. Willatzen, and O. Albrektsen, Detuned electrical dipoles for plasmonic sensing, Nano Lett. 10, 4571 (2010).

[12] F. Zhang, X. Huang, Q. Zhao, L. Chen, Y. Wang, Q. Li, X. $\mathrm{He}, \mathrm{C}$. Li, and K. Chen, Fano resonance of an asymmetric dielectric wire pair, Appl. Phys. Lett. 105, 172901 (2014).

[13] W. X. Lim and R. Singh, Universal behaviour of high- $Q$ Fano resonances in metamaterials: Terahertz to nearinfrared regime, Nano Converg. 5, 5 (2018).

[14] V. V. Khardikov, E. O. Iarko, and S. L. Prosvirnin, Trapping of light by metal arrays, J. Opt. 12, 045102 (2010).

[15] R. Singh, I. A. Al-Naib, Y. Yang, D. Roy Chowdhury, W. Cao, C. Rockstuhl, T. Ozaki, R. Morandotti, and W. Zhang, Observing metamaterial induced transparency in individual Fano resonators with broken symmetry, Appl. Phys. Lett. 99, 201107 (2011).

[16] R. Parker, Resonance effects in wake shedding from parallel plates: Some experimental observations, J. Sound Vib. 4, 62 (1966).

[17] F. Ursell, Trapping modes in the theory of surface waves, Math. Proc. Cambridge Philos. Soc. 47, 347 (1951).

[18] D. C. Marinica, A. G. Borisov, and S. V. Shabanov, Bound States in the Continuum in Photonics, Phys. Rev. Lett. 100, 183902 (2008).

[19] E. N. Bulgakov and A. F. Sadreev, Bound states in the continuum in photonic waveguides inspired by defects, Phys. Rev. B 78, 075105 (2008).

[20] J. Von Neuman and E. Wigner, Über merkwürdige diskrete Eigenwerte, Phys. Z. 30, 467 (1929).

[21] H. Friedrich and D. Wintgen, Interfering resonances and bound states in the continuum, Phys. Rev. A 32, 3231 (1985). 
[22] R. F. Ndangali and S. V. Shabanov, Electromagnetic bound states in the radiation continuum for periodic double arrays of subwavelength dielectric cylinders, J. Math. Phys. (N.Y.) 51, 102901 (2010).

[23] C. W. Hsu, B. Zhen, J. Lee, S.-L. Chua, S. G. Johnson, J. D. Joannopoulos, and M. Soljacić, Observation of trapped light within the radiation continuum, Nature (London) 499, 188 (2013).

[24] F. Monticone and A. Alú, Embedded Photonic Eigenvalues in 3D Nanostructures, Phys. Rev. Lett. 112, 213903 (2014).

[25] M. Rybin and Y. Kivshar, Supercavity lasing, Nature (London) 541, 164 (2017).

[26] M. A. Belyakov, M. A. Balezin, Z. F. Sadrieva, P. V. Kapitanova, E. A. Nenasheva, A. F. Sadreev, and A. A. Bogdanov, Experimental observation of symmetry protected bound state in the continuum in a chain of dielectric disks, arXiv:1806.01932.

[27] Z. F. Sadrieva, I. S. Sinev, K. L. Koshelev, A. Samusev, I. V. Iorsh, O. Takayama, R. Malureanu, A. A. Bogdanov, and A. V. Lavrinenko, Transition from optical bound states in the continuum to leaky resonances: Role of substrate and roughness, ACS Photonics 4, 723 (2017).

[28] S. T. Ha, Y. H. Fu, N. K. Emani, Z. Pan, R. M. Bakker, R. Paniagua-Domnguez, and A. I. Kuznetsov, Directional lasing in resonant semiconductor nanoantenna arrays, Nat. Nanotechnol., DOI: 10.1038/s41565-018-0245-5 (2018).

[29] Y. Plotnik, O. Peleg, F. Dreisow, M. Heinrich, S. Nolte, A. Szameit, and M. Segev, Experimental Observation of Optical Bound States in the Continuum, Phys. Rev. Lett. 107, 183901 (2011).

[30] M. I. Molina, A. E. Miroshnichenko, and Y.S. Kivshar, Surface Bound States in the Continuum, Phys. Rev. Lett. 108, 070401 (2012).

[31] G. Corrielli, G. Della Valle, A. Crespi, R. Osellame, and S. Longhi, Observation of Surface States with Algebraic Localization, Phys. Rev. Lett. 111, 220403 (2013).

[32] E. A. Bezus, L. L. Doskolovich, D. A. Bykov, and V. A. Soifer, Spatial integration and differentiation of optical beams in a slab waveguide by a dielectric ridge supporting high-Q resonances, Opt. Express. 26, 25156 (2018).

[33] M. V. Rybin, K. L. Koshelev, Z. F. Sadrieva, K. B. Samusev, A. A. Bogdanov, M. F. Limonov, and Y. S. Kivshar, High- $Q$ Supercavity Modes in Subwavelength Dielectric Resonators, Phys. Rev. Lett. 119, 243901 (2017).

[34] L. Fonda, Bound states embedded in the continuum and the formal theory of scattering, Ann. Phys. (N.Y.) 22, 123 (1963).

[35] C. S. Kim, A. M. Satanin, Y. S. Joe, and R. M. Cosby, Resonant tunneling in a quantum waveguide: Effect of a finite-size attractive impurity, Phys. Rev. B 60, 10962 (1999).

[36] B. Zhen, C. W. Hsu, L. Lu, A. D. Stone, and M. Soljaĉić, Topological Nature of Optical Bound States in the Continuum, Phys. Rev. Lett. 113, 257401 (2014).

[37] See Supplemental Material at http://link.aps.org/ supplemental/10.1103/PhysRevLett.121.193903 for details of the rigorous derivation of the classical Fano formula for the transmission coefficient of a metasurface; the derivation of Eqs. (2) and (3); a summary of the results for the eigenmode and reflection spectra as functions of the asymmetry parameter $\alpha$ for the cases shown in Figs. 1(b)-1(f); a comparison of the analytical approach of Fig. 4 with numerical simulations based on the realistic dispersion of silicon; and the calculation of the BIC topological charge, which includes Refs. [38-41].

[38] F. Alpeggiani, N. Parappurath, E. Verhagen, and L. Kuipers, Quasinormal-Mode Expansion of the Scattering Matrix, Phys. Rev. X 7, 021035 (2017).

[39] A. B. Evlyukhin, C. Reinhardt, A. Seidel, B. S. Lukyanchuk, and B. N. Chichkov, Optical response features of Si-nanoparticle arrays, Phys. Rev. B 82, 045404 (2010).

[40] J. S. T. Gongora, G. Favraud, and A. Fratalocchi, Fundamental and high-order anapoles in all-dielectric metamaterials via Fano-Feshbach modes competition, Nanotechnology 28, 104001 (2017).

[41] A. B. Evlyukhin, T. Fischer, C. Reinhardt, and B. N. Chichkov, Optical theorem and multipole scattering of light by arbitrarily shaped nanoparticles, Phys. Rev. B 94, 205434 (2016).

[42] M. F. Limonov, M. V. Rybin, A. N. Poddubny, and Y. S. Kivshar, Fano resonances in photonics, Nat. Photonics 11, 543 (2017).

[43] C. Blanchard, J. P. Hugonin, and C. Sauvan, Fano resonances in photonic crystal slabs near optical bound states in the continuum, Phys. Rev. B 94, 155303 (2016).

[44] T. Weiss, M. Mesch, M. Schferling, H. Giessen, W. Langbein, and E. A. Muljarov, From Dark to Bright: First-Order Perturbation Theory with Analytical Mode Normalization for Plasmonic Nanoantenna Arrays Applied to Refractive Index Sensing, Phys. Rev. Lett. 116, 237401 (2016).

[45] J. Lee, B. Zhen, S.-L. Chua, W. Qiu, J. D. Joannopoulos, M. Soljaĉić, and O. Shapira, Observation and Differentiation of Unique High- $Q$ Optical Resonances Near Zero Wave Vector in Macroscopic Photonic Crystal Slabs, Phys. Rev. Lett. 109, 067401 (2012).

[46] E Pshenay-Severin, A. Chipouline, J. Petschulat, U. Hübner, A. Tünnermann, and T. Pertsch, Optical properties of metamaterials based on asymmetric double-wire structures, Opt. Express 19, 6269 (2011).

[47] C. Wu, N. Arju, G. Kelp, J. A. Fan, J. Dominguez, E. Gonzales, E. Tutuc, I. Brener, and G. Shvets, Spectrally selective chiral silicon metasurfaces based on infrared Fano resonances, Nat. Commun. 5, 3892 (2014).

[48] A. Jain, P. Moitra, T. Koschny, J. Valentine, and C. M. Soukoulis, Electric and magnetic response in dielectric dark states for low loss subwavelength optical meta atoms, Adv. Opt. Mater. 3, 1431 (2015).

[49] Y. Zhang, W. Liu, Z. Li, Z. Li, H. Chang, S. Chen, and J. Tian, High-quality-factor multiple Fano resonances for refractive index sensing, Opt. Lett. 43, 1842 (2018).

[50] V. Liu, M. Povinelli, and S. Fan, Resonance-enhanced optical forces between coupled photonic crystal slabs, Opt. Express 17, 21897 (2009).

[51] A. Jain, P. Tassin, T. Koschny, and C. M. Soukoulis, Large Quality Factor in Sheet Metamaterials Made from Dark Dielectric Meta-Atoms, Phys. Rev. Lett. 112, 117403 (2014).

[52] J. Hu, T. Lang, Z. Hong, C. Shen, and G. Shi, Comparison of Electromagnetically-induced transparency performance in metallic and all-dielectric metamaterials, J. Lightwave Technol. 36, 2083 (2018).

[53] S. I. Bozhevolnyi, A. B. Evlyukhin, A. Pors, M. G. Nielsen, M. Willatzen, and O. Albrektsen, Optical transparency by detuned electrical dipoles, New J. Phys. 13, 023034 (2011). 